\title{
«Le sujet âgé » Spécificités odonto-stomatologiques et examen clinique
}

\section{RÉSUMÉ}

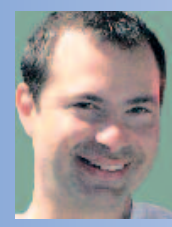

Michel RUQUET

Ancien AHU

Service d'Odontologie

de I'Hôpital Nord (Marseille).

Unité Mixte de Recherche 6578

d'Anthropologie BioCulturelle

(Centre National de la Recherche Scientifique,

Université de la Méditerranée,

Etablissement français du Sang),

UFR d'Odontologie,

27 boulevard Jean-Moulin

13005 Marseille.

Olivier HÜE

PU-PH

Responsable de l'unité fonctionnelle

d'Odontologie restauratrice

Service d'Odontologie Gaston-Berger,

UFR d'Odontologie,

27 boulevard Jean-Moulin

13005 Marseille.

\section{Alain TOSELLO}

MCU-PH

Service d'Odontologie

de l'Hôpital Nord (Marseille).

UFR d'Odontologie.

27 boulevard Jean-Moulin

13005 Marseille.

Le vieillissement physiologique, les pathologies chroniques et I'environnement psychosocial sont source de vulnérabilité chez le sujet âgé. Ces facteurs impactent les pathologies bucco-dentaires alors que les affections orales peuvent à l'inverse les aggraver et conduire le patient dans la spirale de la décompensation. L'examen clinique doit consister en une approche globale de la personne âgée, ce qui lui confère son caractère spécifique. Dans le cadre de cet article, nous proposons une méthodologie d'enquête diagnostique mettant en évidence la nécessité de collaboration interdisciplinaire entre les intervenants médicaux.

- population vieillissante

- affections chroniques

- examen clinique

- approche globale 


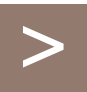

La proportion des sujets âgés (plus de 60 ans) ne cesse de croître en France. Si les tendances démographiques de la période récente se confirment, cette classe d'âge comptera 17 millions de personnes en 2020 et 22,4 millions en 2050. (INSEE site Internet : www.insee.fr). Ce vieillissement inéluctable de la population française a des implications en santé publique et présente un coût pour I'Assurance vieillesse et l'Assurance maladie que la collectivité devra prendre en charge.

Cependant, la notion de vieillissement s'est profondément transformée, ne serait-ce que dans son vocabulaire ; on parle de " séniors " plutôt que de personne âgée. Mais comme l'écrivait Jules Romain dans Knock : " La santé est un état précaire qui ne présage rien de bon ». En réponse à cette prophétie, Ettinger [1] a défini trois catégories de personnes dites " âgées" :

- les patients âgés autonomes ;

- les patients âgés fragilisés ;
- les patients âgés totalement dépendants.

Cette classification est essentiellement basée sur l'accès aux soins et sur les capacités physiques à se déplacer. Elle n'intègre pas la composante psychologique telle que la prend en compte I'International Classification of Fonctionnal, Disability and Health (ICF). La santé et la maladie peuvent être considérées comme un phénomène complexe non linéaire, tortueux avec des conséquences psychologiques bénéfiques ou négatives [2]. Son modèle modifié décrit l'interaction dynamique entre une personne et son environnement en tenant compte des capacités personnelles et des performances dans l'activité journalière ainsi que sa participation à la vie en société (fig. 1). Il s'agit d'un modèle " existentiel » d'évaluation de la santé buccale qui regroupe 12 paramètres (nutrition, attentes du patient, influence de l'environnement social, ressenti et vécu...).

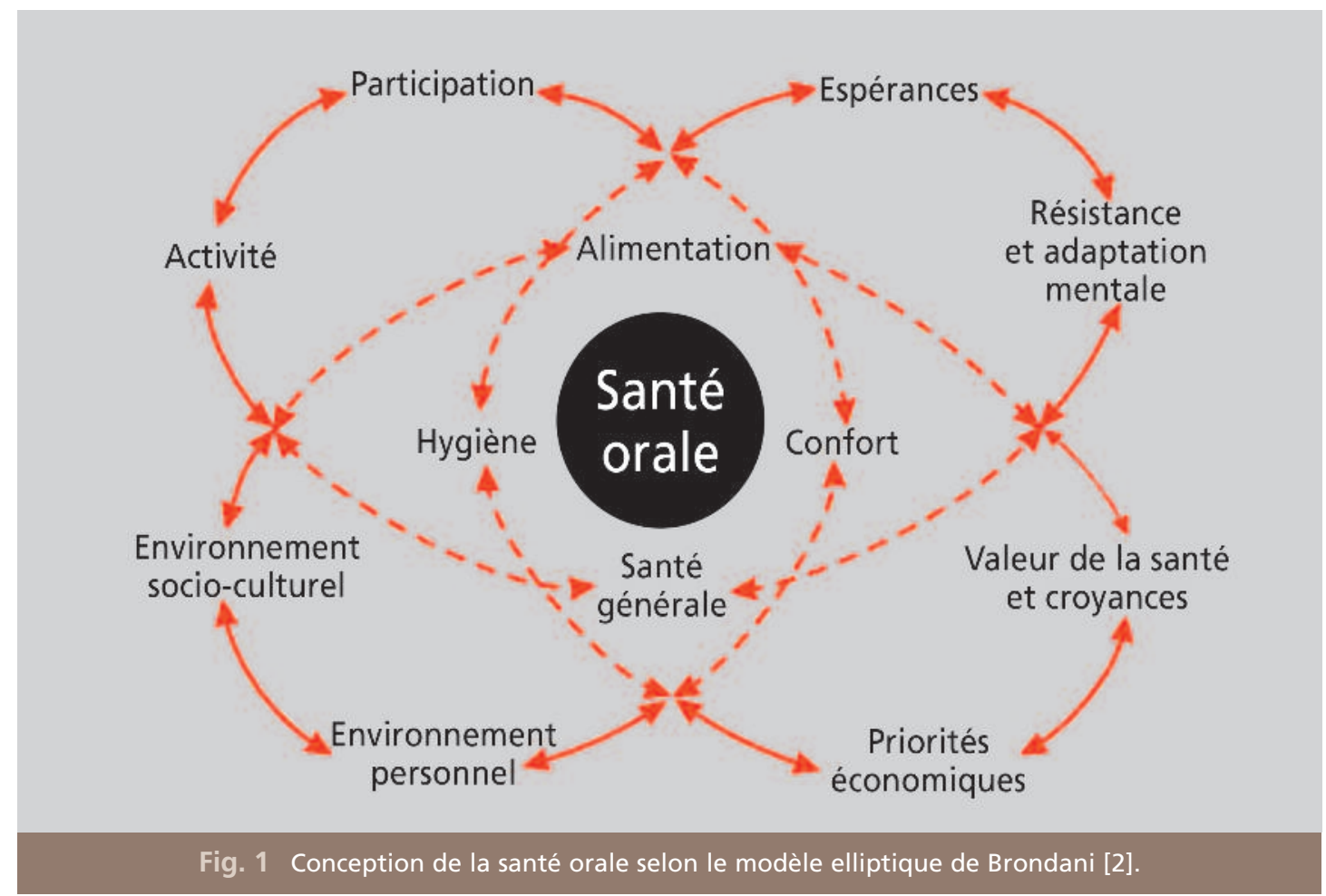


À ce phénomène s'ajoutent les problèmes de santé qui, insidieusement ou de manière plus violente, touchent ces patients [3]. Au niveau de la cavité buccale et plus particulièrement des dents, la situation clinique devient encore plus complexe voire inextricable. L'état de santé général influence l'état buccodentaire qui influence à son tour directement l'état de santé. Ce mécanisme s'accroit au fur et à mesure du passage du patient d'une situation de pleine santé à un état de dépendance marquée.
La connaissance des spécificités des patients fragilisés et dépendants conduit à un examen clinique adapté et permet de répondre aux besoins et aux attentes des malades et de leur famille. Les traitements adéquats sont ainsi effectués dans des conditions de sécurité et de confort optima. Dans le cadre de cet article, un questionnaire médical et bucco-dentaire à destination des praticiens et du personnel soignant des établissements de long séjour est proposé. La synthèse clinique qu'il autorise conduira à un protocole de soins adapté.

\section{Pathologies générales du sujet âgé}

Le nombre de pathologies générales varie avec l'âge quel que soit le niveau de dépendance du patient. L'étude de Denton [3] montre que $90 \%$ des plus de 65 ans souffrent d'une pathologie chronique. Cette enquête s'appuie sur une liste de 32 affections que l'institut de statistiques canadien définit comme chroniques. Parmi celles-ci, les maladies de I'appareil locomoteur et cardiovasculaire ont six fois plus de risques d'être présentes chez les plus de 80 ans que chez les 30/49 ans. Les pathologies neuropsychiatriques, endocriniennes et gastro-intestinales ont elles aussi un taux de prévalence supérieur à 1 chez les sujets les plus âgés. Le tableau I met en évidence que trois quarts des sujets de moins de 65 ans ne présentent aucune pathologie ou seulement une, alors que plus de trois quarts du groupe des plus de 80 ans en avaient deux ou plus.

On comprend, dès lors, que l'âge représente en lui-même un risque pour le praticien de se trouver confronté à une pathologie chronique lors de traitements odontologiques.

Les diverses pathologies rencontrées dans la population étudiée ont des répercussions sur l'état de santé bucco-dentaire et les protocoles de soins et inversement.

Les pathologies gastro-intestinales se manifestent le plus couramment par des douleurs épi-

Tableau I Distribution de la population de l'étude Denton par nombre de pathologies chroniques relevées et classes d'âge (en pourcentage), 2005.

\begin{tabular}{|c|c|c|c|c|c|c|c|c|c|c|}
\hline \multirow{2}{*}{$\begin{array}{l}\text { Classes } \\
\text { d'âge }\end{array}$} & \multicolumn{10}{|c|}{ Nombre de pathologies } \\
\hline & 0 & 1 & 2 & 3 & 4 & 5 & 6 & 7 & $<2$ & $>2$ \\
\hline $12-29$ & 45,5 & 27,7 & 15 & 6,4 & 3 & 1,3 & 0,5 & 0,6 & 73,2 & 26,8 \\
\hline $30-49$ & 35,2 & 28,4 & 16,6 & 8,9 & 5 & 2,7 & 1,2 & 1,9 & 63,6 & 36,3 \\
\hline $50-64$ & 20,7 & 24,7 & 20,3 & 13,5 & 8,6 & 5 & 2,9 & 4,4 & 45,4 & 54,7 \\
\hline $65-79$ & 10 & 18,6 & 20,3 & 17,4 & 13 & 8,8 & 4,6 & 7,3 & 28 & 71,4 \\
\hline $80+$ & 6,7 & 14,9 & 18,1 & 18,2 & 14,9 & 10,4 & 6,9 & 9,9 & 21,6 & 78,4 \\
\hline
\end{tabular}


gastriques, des régurgitations gastro-œsophagiques, des ulcères et des hernies hiatales. Les pathologies digestives spécifiques du sujet âgé n'existent pas mais leur fréquence tend à augmenter avec l'âge. C'est le cas des formations néoplasiques, des diverticuloses chroniques et des lithiases biliaires.

- La plupart des pathologies de ce type se manifestent par des changements muqueux et parodontaux (par exemple, candidoses, douleurs sous prothétiques). Des ulcérations buccales peuvent apparaître dans les cas de lupus, de pemphigus ou de maladies de Crohn. Les lupus se manifestent, également, par des plaques blanches en nid d'abeille, élevées ou kératinisées. Lors de reflux gastroœsophagiens, les troubles boulimiques ou anorexiques sont aggravés. La modification du $\mathrm{pH}$ salivaire rend les abrasions de l'émail fréquentes par attaque acide [4].

- Les pathologies bucco-dentaires peuvent à I'opposé influencer l'évolution des pathologies intestinales. Des études cliniques mettent en évidence une corrélation statistique entre la fréquence d'infections bucco-dentaires, d'Helicobacter pylori et la présence d'une infection de la muqueuse gastrique [5].

Les pathologies cardio-pulmonaires sont observées sous toutes leurs formes: hypertension artérielle, insuffisance cardiaque, séquelles d'accidents vasculaires cérébraux. L'étude Framingham [6] montre, par exemple, que l'incidence annuelle de l'insuffisance cardiaque augmente de façon exponentielle avec l'âge : 3/1 000 chez les 35-64 ans, 10/1 000 chez les 6574 ans avec une explosion dans la décennie 8594 ans. La prévalence de l'HTA systolique isolée varie entre 20 à $80 \%$ alors que la moyenne tous âges confondus est de $11 \%$. La pression artérielle systolique s'élève également de façon linéaire jusqu'à 80/90 ans alors que la pression artérielle diastolique croît jusqu'à 50 ans puis diminue.

- Ces pathologies nécessitent une prise en charge bucco-dentaire d'urgence afin de limiter les surinfections. Elles peuvent conduire lorsqu'elles sont en évolution à des extractions multiples afin d'éradiquer les foyers infectieux avérés. Une hypertension artérielle non traitée diminue la résistance au stress et prédispose à des accidents au fauteuil allant de la simple angoisse à l'infarctus.

- Les infections bucco-dentaires peuvent aggraver une pathologie valvulaire. Le passage des germes à l'intérieur de la circulation sanguine, d'une manière soit spontanée c'est-àdire en provenance d'un foyer infectieux, soit provoquée par des manipulations utilisant des instruments souillés, aboutit à une greffe bactérienne sur les valves cardiaques. II peut s'agir par exemple d'un rétrécissement ou d'une insuffisance de fonctionnement de la valve aortique, voire d'une insuffisance de fonctionnement de la valve mitrale [7].

Certaines études annoncent une relation entre infection périapicale et atteintes d'athérosclérose. II semblerait exister un lien causal entre parodontites et athérosclérose dont le mécanisme supposé est l'invasion bactérienne des artères [8].

Les pathologies neuropsychiatriques telles les délires obsessionnels, les cas de maladie de Parkinson et de maladie d'Alzheimer ont une prévalence qui tend à s'accroître avec l'allongement de la durée de la vie. Dans le cadre de l'étude PAQUID [9] ${ }^{(1)}$, il apparaît que la préva-

(1) Etude PAQUID : Epidémiologie de la maladie d'Alzheimer, des syndromes apparentés, de la dépendance et des affections liées au vieillissement. 
lence globale de la démence est de $4,3 \%$ de la population de plus de 65 ans et celle de la maladie d'Alzheimer est de 3,1\%. Pour cette dernière, elle est de $1,8 \%$ pour les sujets vivant à domicile et de $26,3 \%$ en institution [10]. Chez nos aînés isolés socialement et mis à l'écart de leur cellule familiale, les manifestations de démence sénile sont amplifiées.

- Ces pathologies ont des répercussions buccodentaires majeures. Les polymédications, sialoprives pour la plupart, prédisposent à des atteintes carieuses nombreuses. Les problèmes neurologiques de type sénilité, démence, maladie d'Alzheimer et psychiques (dépression) compliquent la communication patient/praticien, les capacités d'adaptation et le maintien de l'hygiène. Ils aggravent les manifestations locales citées ainsi que les troubles de l'alimentation [11].

- La perte des dents peut conduire à une dégradation de l'image du patient, favorisant son isolement et aggravant, ainsi, les syndromes dépressifs et les perturbations alimentaires.

Les pathologies endocriniennes fréquemment observées sont généralement des diabètes de type I ou II, accompagnés d'une alimentation trop sucrée ou au contraire carencée. La prévalence des diabètes chez les personnes âgées vivant en institution est de 8,5\% [12]. Cet état est une des causes d'apparition de caries, en particulier du collet, des problèmes de nutrition. Par rapport aux sujets sains du même âge, il dégrade les conditions de vie.

- Mal équilibré le diabète conduit à des extractions de dents pathologiques en prévention du risque infectieux. Il diminue les capacités de cicatrisation et la salive riche en glucose prédispose aux polycaries.

- Un traitement antibiotique par sulfamide lors d'une infection dentaire peut, chez ces patients, augmenter le risque hypoglycé- miant de même qu'une infection buccale non traitée peut déséquilibrer le diabète.

Les restrictions locomotrices découlent, entre autres, d'accidents vasculaires cérébraux ou de séquelles de fractures. Ces pathologies ont un retentissement sur l'état général : hypoventilation, diminution de l'oxygène circulatoire, asthénie. Elles amoindrissent l'effort physique et ont un effet néfaste sur l'appétit.

- Elles provoquent, la plupart du temps, un retard d'accès aux soins face à l'impossibilité de déplacement au cabinet dentaire. S'il existe une atteinte des membres supérieurs, la préhension et les mouvements nécessaires au brossage sont difficiles. L'hygiène en sera affectée. Les pathologies rhumatismales de type polyarthrite engendrent les mêmes effets.

- Les infections orales bactériennes et l'inflammation semblent, à l'inverse, impacter directement l'évolution négative des polyarthrites chroniques évolutives [13].

Chez les personnes âgées, la dégénérescence physiologique touche la plupart des organes fonctionnels. Au niveau de la cavité buccale, cette dégénérescence se traduit par des altérations des fonctions telles que la gustation, par une réduction des capacités d'adaptation, par diminution du potentiel de cicatrisation.

L'ensemble des tissus constituant l'organe dentaire subit un processus de sénescence qui limite la capacité de réparation de l'organe dentaire. La rétraction physiologique du parodonte favorise l'exposition radiculaire. La résistance des collets au développement des lésions carieuses est amoindrie. Ce risque carieux constitue une pathologie spécifique et leur prévalence augmente de 18 à $51 \%$ [14]. Les pathologies pulpaires évoluent habituellement à bas bruit ; à partir de dents nécrosées, elles entraînent des foyers infectieux latents. Ces foyers 
peuvent progresser sur des durées relativement longues sous forme de lésions apicales et développent des pertes osseuses importantes.

Les changements physiologiques des tissus parodontaux réduisent leur potentiel de défense et de cicatrisation. 55 à $85 \%$ des patients présenteraient une maladie parodontale par altération des réponses immunitaires, par diminution des capacités de remodelage et de cicatrisation des tissus ainsi qu'une prolifération des micro-organismes pathogènes liés à une diminution $\mathrm{du} \mathrm{pH}$. Les muqueuses buccales deviennent, elles aussi, atrophiques. L'atrophie muqueuse entraîne une susceptibilité accrue aux traumatismes et une intolérance fréquente au port de prothèses dentaires [15]. Outre les ulcérations et les lésions traumatiques, les candidoses et les brûlures se rencontrent souvent dans cette population. Ces lésions se localisent préférentiellement au niveau des plaques bases prothétiques. Des perlèches, des hyperplasies muqueuses, des lichens et des kératoses sont aussi observés. Au niveau de la muqueuse linguale, l'involution des papilles gustatives, impliquées dans la per- ception du goût et des saveurs sont à l'origine de la diminution de l'appétit.

La diminution de la sécrétion salivaire liée au vieillissement est peu importante mais la prise de médicaments contribue de manière majeure à cette diminution. L'étude d'Ichikawa [16] chez des sujets de plus de 79 ans vivant à domicile montre que les patients se plaignant de sècheresse buccale prennent plusieurs médicaments et que le volume salivaire a tendance à diminuer proportionnellement. Le ralentissement de la fonction rénale et le faible niveau de métabolisme expliquent l'élimination plus lente des agents actifs avec une potentialisation de leurs effets. Les médicaments cardiovasculaires (antihypertenseurs et diurétiques) entrainent une réduction du flux sanguin périphérique dont la conséquence est une diminution de la quantité de salive. Les médicaments pour le diabète et l'ulcère gastro-intestinal produisent les mêmes résultats. Les hypnotiques et les anxiolytiques sont, également, une cause d'altération de la fonction salivaire et du sentiment de sécheresse buccale. Cette hyposialie

\section{Tableau II Conduite à tenir en fonction des affections chroniques.}

\begin{tabular}{|c|c|c|}
\hline Type de pathologie & Syndrome clinique & C.A.T. \\
\hline Pathologies gastro-intestinales & $\begin{array}{l}\text { - perturbation alimentaire } \\
\text { - érosions dentaires }\end{array}$ & $\begin{array}{l}\text { - surveillance alimentaire, conseils diététiques } \\
\text { - traitements dentaires conservateurs }\end{array}$ \\
\hline Pathologies cardio-pulmonaires & $\begin{array}{l}\text { - HTA } \\
\text { - pathologies valvulaires } \\
\text { - risque hémorragique } \\
\text { - sensation de fatigue } \\
\text { - ATCD infarctus }\end{array}$ & $\begin{array}{l}\text { - maîtrise nervosité ; AINS }<15 \mathrm{j} \\
\text { - couverture } A B \text { préventive } \\
\text { - arrêt AAP et pas d'arrêt répétés AVK } \\
\text { - protocoles de soins adaptés } \\
\text { - pas de vasoconstricteurs }\end{array}$ \\
\hline Pathologies neuro-psychiatriques & $\begin{array}{l}\text { - xérostomies } \\
\text { - anorexie/dépression } \\
\text { - syndromes parkinsoniens }\end{array}$ & $\begin{array}{l}\text { - prise en charge par substituts salivaires } \\
\text { - conseils alimentaires + surveillance } \\
\text { - Cl macrolides }\end{array}$ \\
\hline Pathologies endocriniennes & - xérostomies & - contrôle glycémie à jeun, $\mathrm{Cl}$ corticoïdes et AINS \\
\hline Pathologies locomotrices & $\begin{array}{l}\text { - hygiène déficiente } \\
\text { - problème de nutrition }\end{array}$ & $\begin{array}{l}\text { - matériel adapté au handicap } \\
\text { - conseils diététiques }\end{array}$ \\
\hline
\end{tabular}


provoque des manifestations fonctionnelles qui renforcent non seulement le risque de carie dentaire et de maladie parodontale, mais peut aussi causer une difficulté à parler ou à déglutir. La sensation de goût est altérée et accompagnée le plus souvent de sensation de brûlure et d'halitose. Les conséquences de la dégénérescence physiologique des structures buccales sont la plupart du temps des phénomènes de dénutrition et d'abandon du port des prothèses; les pathologies générales existantes sont alors accentuées.
Les pathologies générales provoquent des retards d'accès aux soins et compliquent les affections bucco-dentaires qui en découlent. Leur diagnostic précoce se fait en association avec les spécialistes concernés lorsqu'il existe des manifestations buccales et évite leur aggravation.

Les conduites à tenir dans l'exercice de l'odontologie gériatrique sont adaptées à chacune des pathologies systémiques connues ou diagnostiquées et résumées dans le tableau ci-dessus (tableau II) [11].

\section{Détermination du profil nutritionnel}

La pathologie de la nutrition est en constante augmentation avec le vieillissement de la population. Elle est une cause majeure d'accroissement de la morbidité et de la mortalité chez les plus de 60 ans. Grâce au traitement de leurs manifestations bucco-dentaires et aux conseils diététiques qu'il peut prodiguer, le chirurgien-dentiste a un rôle important à jouer dans le domaine du dépistage et de la prévention des troubles de l'alimentation [17-19].

La malnutrition a deux causes principales :

- une cause endogène par hypercatabolisme (pathologies générales et syndromes infectieux) ;

- une cause exogène par insuffisance d'apport alimentaire.

En conséquence, l'examen clinique et l'anamnèse doivent, en dehors des données classiques, porter sur les éléments de santé générale préalablement cités. La présence de pathologies psychiatriques graves, de pathologies gastrointestinales (dérèglement de l'absorption intestinale), d'insuffisances cardio-respiratoires où le fait de s'alimenter demande un effort (dys- pnée d'effort) ainsi que les régimes alimentaires stricts doivent alerter sur le risque de dénutrition. Il convient d'être également vigilant sur les conditions de vie du patient. Les hospitalisations en long séjour, en réanimation, l'isolement et les conditions économiques faibles sont des facteurs de disparition des prises de repas structurées. Ces patients ont tendance à multiplier les collations, avec une alimentation souvent sucrée, grasse, pauvre en protéines, vitamines et fibres.

Le chirurgien-dentiste peut se référer à des examens simples de détermination de la dénutrition : I'examen pondéral, l'examen clinique, l'évaluation biologique et le test MNA (Mini Nutritional Assessment ${ }^{\odot}$ ). Le calcul de l'indice de masse corporelle $(19<\mathrm{IMC}<25)$ permet d'évaluer simplement la perte pondérale. L'importance et la vitesse de cette perte doivent faire penser à un problème de nutrition. La mesure des plis cutanés et de la circonférence brachiale sont aussi à considérer. Sur le plan clinique, certains signes de dénutrition sont facilement décelables par le praticien : les chéilites, les lésions gingivo-dentaires et l'aspect dépa- 
pillé de la langue. Lors de l'interrogatoire du patient, les questions portent sur la présence de tachycardies, de dyspnées d'effort, de vomissements, de diarrhées et de douleurs abdominales. Le dosage de la pré-albumine et plus couramment de l'albumine confirme un trouble de l'alimentation ( $\mathrm{Tx}$ albumine $<35 \mathrm{~g} / \mathrm{l}$ ). Le test MNA est une méthode simple d'évaluation du niveau nutritionnel d'une personne âgée.

L'examen endobuccal fait apparaître des pathologies locales spécifiques aux troubles de l'alimentation, un état dento-prothétique dégradé et des habitudes alimentaires initiatrices d'un état de dénutrition (fig. 2 à 4). La

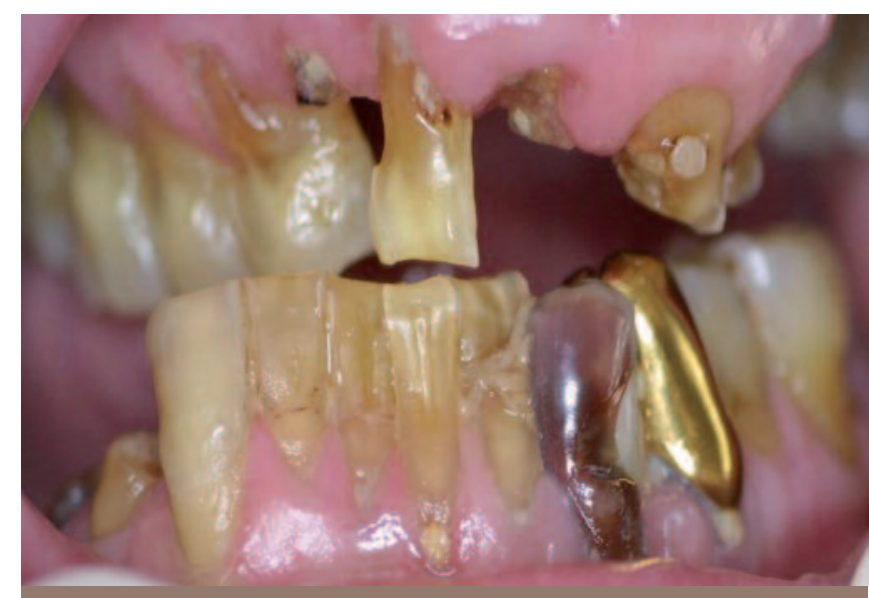

Fig. 2 État bucco-dentaire aggravé ; insuffisance des couples dentaires antagonistes. plaque dentaire et la mauvaise hygiène buccodentaire agissent, semble-t-il, comme un réservoir de pathogènes respiratoires, particulièrement chez des patients atteints de maladie parodontale [20]. L'insuffisance masticatoire est consécutive à un édentement important ou à des réhabilitations prothétiques inadaptées : douleurs sous-prothétiques, brûlures des muqueuses, instabilité des bases.

L'abandon de la prothèse ou la modification des cycles masticatoires peuvent se traduire par des sélections alimentaires avec pour conséquences des carences nutritionnelles et une insuffisance quantitative des rations.
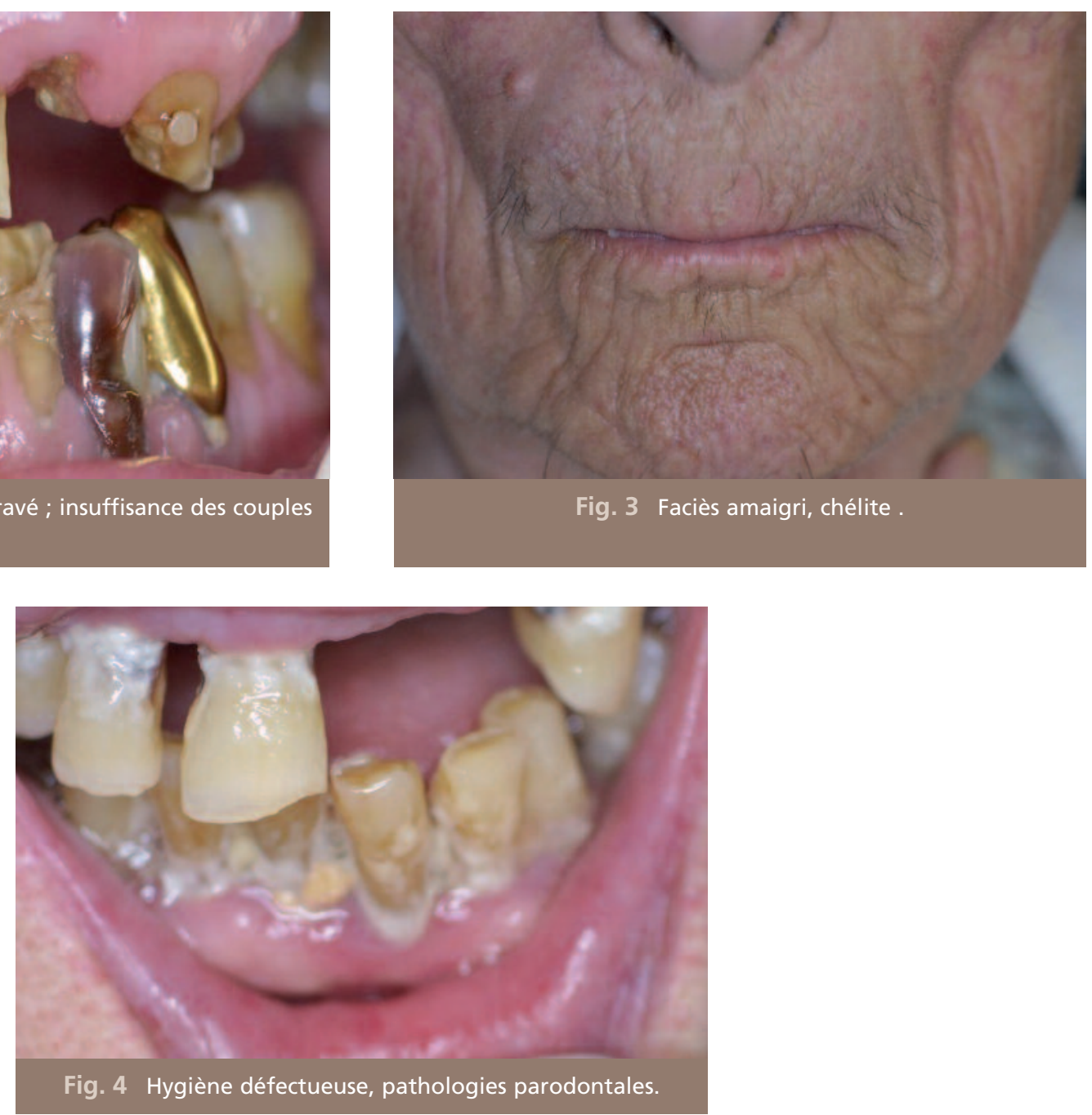
Le rétablissement de la fonction masticatrice diminue le risque de dénutrition et évite l'aggravation des pathologies générales. II convient dans un premier temps d'éradiquer les douleurs, puis dans un deuxième temps de réaliser très rapidement la réhabilitation fonction- nelle. Elle est complétée par une rééducation alimentaire, capable d'apporter protéines, glucides, oligo-éléments et vitamines. Les conseils diététiques sont du ressort du praticien traitant qui, dans les cas sévères, peut collaborer avec un médecin nutritionniste (fig. 5).

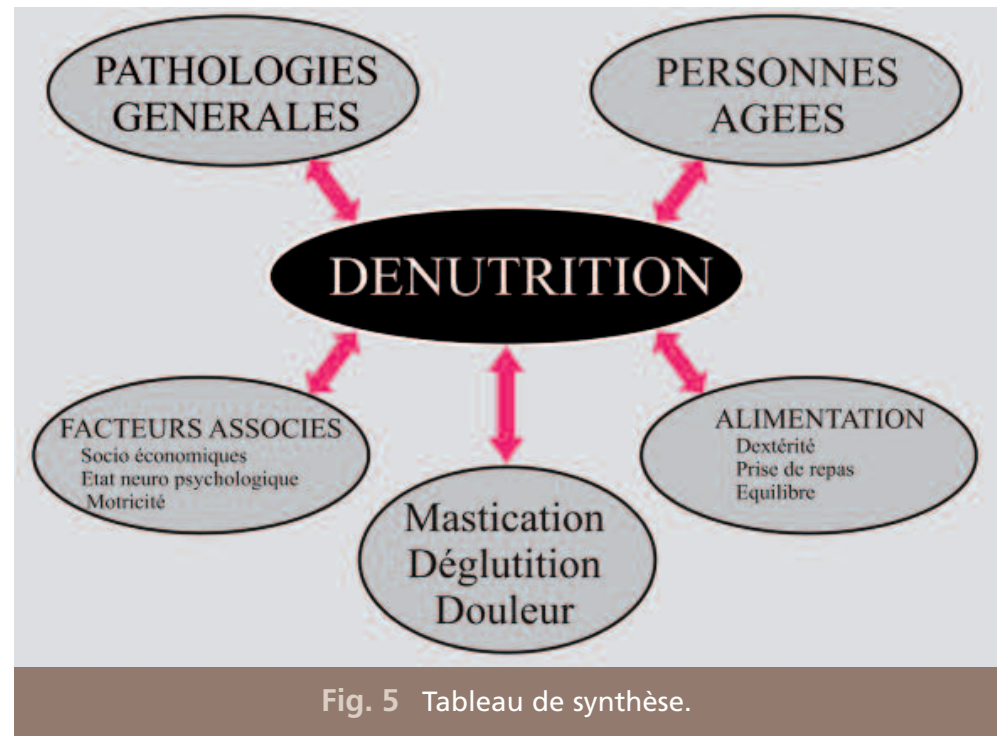

\section{Facteurs socio-économiques}

\section{et comportementaux}

De nombreuses études [21, 22, 23] montrent un lien entre statut économique et état de santé. Les retraités sont souvent plus exposés aux inégalités sociales que les sujets en activité ; cela influe sur leur niveau de santé bucco-dentaire. En effet, les différences d'exposition aux facteurs de risque, de comportements (hygiène, alimentation) et de disparités $d$ 'accès aux soins varient en fonction du niveau socio-économique.

Le niveau d'étude a un impact sur l'état de santé et sur le recours aux soins. Les personnes à faible niveau d'éducation déclarent plus de problèmes dentaires, plus de prothèses amovibles et moins de prévention. Le lien avec le niveau de revenu va dans le même sens. Les demandes en prothèse fixée sont plus importantes dans les catégories socio-économiques élevées (rapport avec le niveau de revenu) alors que celles en extraction sont plus prépondérants dans les groupes à faible niveau socio-professionnel. Les stratégies d'accès aux traitements prothétiques diffèrent donc selon le niveau de ressources matérielles. Les personnes à bas revenus sont donc exposées à un « phénomène de renoncement » aux soins. 
Les habitudes et les comportements individuels sont tout autant en relation avec la santé bucco-dentaire. Le tabagisme, les troubles de la dénutrition et la mauvaise hygiène sont des facteurs de risque importants pour les pathologies parodontales et les lésions carieuses.

Les patients isolés socialement, en hospitalisation de long séjour ou en institution, montrent habituellement une hygiène déficiente, un état de malnutrition et ils accèdent difficile- ment aux soins. Ces déterminants ont une incidence importante sur leur santé bucco-dentaire.

En conséquence, chez tout patient dont l'état de santé bucco-dentaire est fortement dégradé, le praticien se doit de soupçonner un ou plusieurs des facteurs préalablement exposés. Le rôle de prévention du chirurgien-dentiste consiste en une collaboration entre services compétents.

\section{Conduite pratique de l'examen clinique}

Lors de la première consultation ou dès l'arrivée du patient en institution, le chirurgiendentiste conduit conjointement aux autres spécialistes gériatres l'anamnèse médicale ainsi qu'une évaluation des facteurs environnementaux et comportementaux. II cerne ses besoins et ses impératifs.

Le praticien relève le sexe, l'âge, les pathologies générales en évolution, les antécédents médicaux connus ainsi que la liste et la posologie des médicaments prescrits. II note le niveau de dépendance et les altérations psychologiques. Pour cette évaluation, une grille nationale standardisée est utilisée. Le degré de perte d'autonomie est défini par un questionnaire, qui détermine son Groupe Iso Ressource " GIR ». Cette donnée sert à déterminer le montant de I'Allocation personnalisée d'autonomie.

L'examen bucco-dentaire se fait à la fois sur le plan exo-buccal et endo-buccal. Dans certains cas cliniques, l'examen exo-buccal ne reflète pas un état bucco-dentaire dégradé ; un examen endo-buccal, réalisé par un spécialiste, est alors indispensable. $\mathrm{Si}$, pour des raisons d'éloigne- ment géographique, la présence régulière d'un chirurgien-dentiste est impossible, il est possible d'avoir recours à des caméras endo-buccales permettant des consultations à distance.

L'odontologiste vérifie l'état fonctionnel des prothèses et détermine le besoin en soins prothétiques du patient. La demande de celui-ci est parfois contraire aux désirs des accompagnants ou des personnes référentes.

Le questionnaire se fait idéalement, en présence du patient et du personnel soignant. II met en évidence le niveau d'hygiène, le suivi dentaire, le niveau de sensibilité gustative, olfactive et les habitudes alimentaires.

La présence d'une aide pour l'hygiène et la prise des repas est un atout pour les sujets âgés dépendants et doit être systématiquement demandée.

L'évaluation nutritionnelle tient compte de l'aspect biométrique précédemment cité ainsi que de l'interprétation du test MNA et du dosage biologique de l'albumine.

Une fiche type est proposée aux praticiens et aux établissements lors de l'entrée d'un 


\section{NOM :}

NOM DE JEUNE FILLE :

PRENOMS :

DATE DE NAISSANCE :

POIDS :

TAILLE :

\section{RENSEIGNEMENTS MEDICAUX}

Pathologies antérieures connues?

OUI $\square$ NON $\square$

Si oui lesquelles?

Pathologies actuelles?

OUI $\square$ NON $\square$

Si oui lesquelles?

Prise en charge dans le cadre d'une ALD (Affection de Longue Durée) ?

Si oui pourquoi ?

Allergies médicamenteuses, alimentaires, autres... ?

OUI $\square$ NON $\square$

OUI $\square$ NON $\square$

Interventions chirurgicales ?

OUI $\square$ NON $\square$

Traitements médicamenteux

\begin{tabular}{|l|l|l|}
\hline MATIN & MIDI & \\
\hline & & \\
& & \\
& & \\
\hline
\end{tabular}

\section{NIVEAU D'AUTONOMIE}

GIR actuel si évaluation réalisée

État sensoriel

Troubles du comportement ?

OUI $\square$ NON $\square$

Communication ? facile $\square$ difficile $\square$ impossible $\square$

Pourquoi ?

\section{Valide}

Semi-valide :

Marche avec

- un déambulateur

- un fauteuil roulant

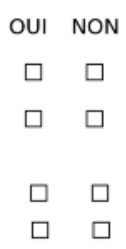

\section{ALIMENTATION}

Mange seul(e) :

OUI $\square$ NON $\square$

- texture normale

OUI NON

- texture hachée

- texture moulinée

Régime alimentaire ?

OUI $\square$ NON $\square$

Lequel ?

Examens complémentaires réalisés ? (MNA, mesures anthropométriques, dosage préalbumine) 


\section{EXAMEN BUCCO-DENTAIRE}

Niveau d'hygiène :

Assure-t-elle seule ses soins bucco-dentaires ?

OUI $\square$ NON $\square$

Présence d'une aide à l'hygiène :

OUI $\square$ NON $\square$

Si oui, nombre de brossages quotidiens :

Indice de plaque :

Scores 0 : aucune plaque,

1 : film de plaque adhérant à la gingivale marginale,

2 : accumulation modérée de dépôts dans la poche gingivale,

3 : abondance de matière dans la poche gingivale et/ou sur la dent et la gencive.

\begin{tabular}{|c|l|l|l|l|l|}
\hline Numéro de la dent & Mésial & Distal & Vestib. & Lingual & Index \\
\hline 16 & & & & & \\
\hline 21 & & & & & \\
\hline 24 & & & & & \\
\hline 36 & & & & & \\
\hline 41 & & & & & \\
\hline 44 & & & & & \\
\hline
\end{tabular}

Hyosialie :

OUI $\square$ NON $\square$

Bilan muco-gingival :

Présence d'une maladie parodontale :

OUI $\square$ NON $\square$

Existence d'une pathologie muqueuse (Candidose)

OUI $\square$ NON $\square$

Existence de blessures:

OUI $\square$ NON $\square$

\section{Bilan prothétique :}

Prothèses existantes :

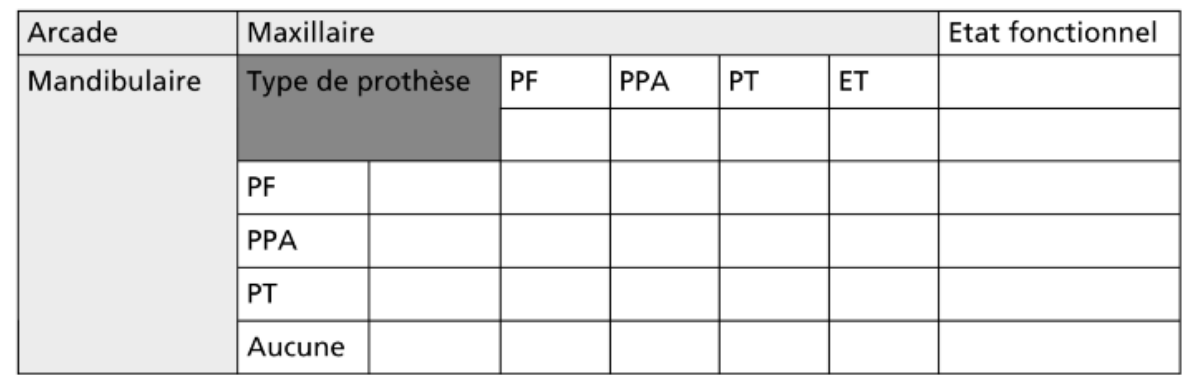

État des prothèses :

Capacité masticatrice : (nombre de couples de dents PM/M présents)

Niveau de satisfaction masticatoire :
- patient
$1 \square 2 \square 3 \square$
- personnel soignant $1 \square 2 \square 3 \square$ 
patient pour un long séjour. Elle permet un relevé systématique des informations médicales nécessaire à l'établissement d'un plan de traitement adapté.

Cette méthodologie met en évidence l'impossibilité de grouper nos patients âgés en une seule catégorie. Elle permet d'apprécier le degré de vieillissement, d'adaptation et d'autonomie.

Certains patients sont demandeurs de soins de qualité, consultent de manière autonome et présentent une hygiène satisfaisante ; les soins sont identiques à ceux réalisés sur des adultes plus jeunes. À l'opposé, il existe des patients plus fragilisés où les soins classiques sont impossibles en raison de polypathologies sévères, de prise de médicaments ou d'une hygiène insuffisante ; les traitements se limite-

\section{Conclusion}

Les problèmes liés aux sujets âgés sont souvent sous-estimés en matière de santé bucco-dentaire.

Le chirurgien-dentiste a un rôle prépondérant dans le diagnostic, la prévention et le traitement des pathologies bucco-dentaires mais également dans les relations entre santé buccale et santé générale. II s'intègre dans une véritable " équipe gériatrique " dont il est un des maillons indispensables [24]. II est également l'interlocuteur privilégié des pouvoirs publics en ce qui concerne le développement de réseaux de soins qui commencent à voir le jour dans plusieurs régions. Par ailleurs, il doit avoir conscience de la particularité des soins liés ront à l'élimination de la douleur, des foyers infectieux et à des traitements prothétiques à minima.

Entre ces deux situations cliniques nous avons à traiter des patients pour lesquels conseils, informations patience et soins adaptés sont indispensables.

La démarche médicale ou odontologique classique recherche une unicité en regroupant les signes en syndromes pour définir une maladie unique. En gériatrie, cet effort d'unicité demeure nécessaire, mais l'enquête diagnostique conclut le plus fréquemment à l'existence d'un état polypathologique. L'établissement d'un plan de traitement à l'issue de l'examen clinique nécessite donc une prise en charge multidisciplinaire et une bonne évaluation bénéfice/risque.

à cette population qui nécessitent compétence, attention et disponibilité. Dans cette perspective, les enseignements dans les facultés et les formations continues sont à privilégier. L'odontologiste conseille les différentes instances sociales, les patients et leur famille sur la nécessité d'une prévention efficace. La politique actuelle de santé publique est axée sur I'intervention (CMU) qui réduit les inégalités mais qui est peu orientée sur la prévention [25]. Développer des consultations préventives chez les plus de 60 ans en association avec d'autres spécialistes gérontologues n'est-il pas la voie d'avenir pour traiter la personne " âgée " dans sa globalité ? 


\section{Bibliographie}

1. Ettinger $\mathrm{WH} \mathrm{Jr}$.

Geriatrics program

development

at Bowman Gray School

of Medicine

of Wake Forest University.

Am J Med 1994

Oct;97(4A):49S-50S.

2. Brondani MA, Bryant SR, MacEntee MI.

Elders assessment of an evolving model of oral health.

Gerodontology

2007 Dec;24(4):189-95.

3. Denton FT, Spencer BG.

Chronic health conditions: changing prevalence in an aging population and some implications for the delivery of health care services. Can J Aging 2010 Mar;29(1):11-21.

4. Chi AC, Neville BW, Krayer JW, Gonsalves WC. Oral manifestations of systemic disease. Am Fam Physician 2010 Dec;82(11):1381-8.

5. Kopaánski Z, Cienciala A, Banaś J, Kamiński B, Witkowska B, Zastepa P, Brandys J, Micherdziński J. Coexistence of infection of the oral cavity and stomach and duodenal mucosa with Helicobacter pylori in patients with ulcer and chronic gastritis. Wien Klin Wochenschr 1995;107(7):219-24.

6. Burt VL, et al. Trends in the prevalence, awareness, treatment, and control of hypertension in the adult US population. Data from the health examination surveys,

1960 to 1991.

Hypertension

1995;26(1):60-9.

7. Warburton $\mathrm{G}$,

Caccamese JF Jr.

Valvular heart disease

and heart failure:

dental management

considerations.

Dent Clin North Am

2006 Oct;50(4):493-512, v.

8. Zoellner $\mathrm{H}$.

Dental infection and vascular disease.

Semin Thromb Hemost 2011 Apr;37(3):181-92. Epub 2011 Mar 31.

9. Dartigues JF, et al. Principal lifetime occupation and cognitive impairment in a French elderly cohort (Paquid).

Am J Epidemiol 1992 May;135(9):981-8.

10. Lobo A, et al. Prevalence of dementia and major subtypes in Europe:

A collaborative study of population-based cohorts. Neurologic Diseases in the Elderly Research Group. Neurology 2000;54(11 Suppl 5):S4-9.

11. Dupuis $V$, Leonard $A$. Odontologie du sujet âgé : spécificités et précautions. Paris : Ed. Elsevrier Masson SAS, 2010.

12. Bourdel-Marchasson I, et al. Prevalence of diabetes and effect on quality of life in older French living in the community: the PAQUID Epidemiological Survey.

J Am Geriatr Soc 1997 Mar;45(3):295-301.
13. Detert J, Pischon N, Burmester GR, Buttgereit F. The association between rheumatoid arthritis and periodontal disease. Arthritis Res Ther 2010;12(5):218. Epub 2010 Oct 22.

14. Bailey $\mathrm{RL}$, et al. Persistent oral health problems associated with comorbidity and impaired diet quality in older adults.

J Am Diet Assoc 2004 August;104(8):1273-6.

15. Ghezzi EM, Ship JA. Systemic diseases and their treatments in the elderly: impact on oral health. $J$ Public Health Dent 2000 Fall;60(4):289-96.

16. Ichikawa $\mathrm{K}$, et al. Relationships between the amount of saliva and medications in elderly individuals. Gerodontology 2011 Jun;28(2):116-20.

17. Miller C, Morvan C, Parmentier P, Lasfargues JJ. Nutrition et santé bucco-dentaire : rôle du chirurgien-dentiste. Revue du Comité National d'Hygiène et Science 2005;3.

18. Tosello A, Niviere $C$, Ruquet M, Andrieu I. La dénutrition chez les personnes âgées. Cah Prothèse 2006 juin;134:41-50.

19. Veyrune JL. Lassauzay $C$, Nicolas $E$, Hennequin M. Considérer le risque de dénutrition chez les personnes âgées. Réal Clin 2004;15(4):361-73. 
20. Scannapieco FA, Papandonatos GD, Dunford RG.

Associations between oral conditions and respiratory disease in a national sample survey population. Ann Periodontol 1998 July;3(1):251-6.

21. Tavitian $P$, et al. État bucco-dentaire et statut nutritionnel : étude anthropoépidémiologique chez les personnes âgées. Antropo 2010;22:19-28.
22. Riley JL, et al. Dental attitudes: proximal basis for oral health disparities in adults.

Community Dent

Oral Epidemiol

2006 August;34(4):289-98.

23. Warmala $S$, et al. Inequity in access to dental care services explains current socioeconomic disparities in oral health: The Swedish National Surveys of Public Health 2004-2005.
J Epidemiology Community Health 2006;60(12):1027-33.

24. Gibert $\mathrm{GH}$, et al. Use of specific dental treatment procedures by dentate adults during a $\mathbf{2 4}$ month period. Community Dent Oral Epidemiol 2002 Aug;30(4):260-76.

25. Auvray L, Dumesnil S, Le Fur $P$.

Santé, soins et protection sociale en 2000 . Rapport Credes, Série et résultats 2001;1364.

\section{SUMMARY}

\section{"The eldely person" Oral specificities and clinical examination}

Michel RUQUET,

Olivier HÜE,

Alain TOSELLO

\section{Keywords \\ - aging population - chronic conditions \\ - clinical examination \\ - global approach}

The physiological ageing, the chronic conditions and the psycho-social environment are source of vulnerability to the old subject. These factors impact on the oral pathologies while the oral affections can deteriorate them on the contrary and lead the patient in the spiral of the decompensation. The clinical examination has to consist of a global approach of the eldely person, what confers it a specific character. Within the framework of this article, we propose a methodology of diagnostic survey bringing to light the necessity of interdisciplinary collaboration between the medical participants. 


\section{LA VIE DE L'ASSOCIATION}

PRÉSIDENTS D'HONNEUR :

Pierre CERNÉA ${ }^{\dagger}$, Jean OUVRARD ${ }^{\dagger}$, Michel BENOIST ${ }^{\dagger}$,

Hubert OUVRARD

\section{MEMBRES D'HONNEUR :}

A. RICHARD ${ }^{\dagger}$, H. LENTULO ${ }^{\dagger}$,

A. LAMBERT, P. GONON ${ }^{\dagger}$,

J.-P. RAGOT'

Ch. DESCROZAILLES ${ }^{\dagger}$,

G. FICHELLE ${ }^{\dagger}$

\section{CONSEIL D'ADMINISTRATION}

PRÉSIDENT : LUC CHIKHANI

VICE-PRÉSIDENT :

Jean-François LEGRAND

SECRÉTAIRE GÉNÉRAL :

Frédéric LARCHÉ

TRÉSORIER :

Gérard PASQUET

MEMBRES :

Danielle BUCH, Jean BUQUET, Estelle FAVRE, Jean MEYER,

Guy PRINC, Valérie TRAVERT

\begin{abstract}
COMITÉ SCIENTIFIQUE
PRÉSIDENT : LUC CHIKHANI

VICE-PRÉSIDENT : Hubert OUVRARD

SECRÉTAIRE : Frédéric LARCHÉ

MEMBRES: Christophe BONNEFOY, Estelle FAVRE, Thierry GUÉRIN, Jean-François LEGRAND, Frank LEVAVASSEUR, Hervé MISSISTRANO, Gérard PASQUET, Thierry PIRAL, Valérie TRAVERT
\end{abstract}

ANCIENS PRÉSIDENTS: P. CERNÉA ${ }^{\dagger}$, P. HENNION, H. LENTULO ${ }^{\dagger}$, M. LEPOIVRE ${ }^{\dagger}$, A. MARMASSE ${ }^{\dagger}$, A. LAMBERT, R.-R. RIGOLET ${ }^{\dagger}$, R. BATAILLE ${ }^{\dagger}$, L.-A. STIEGLER ${ }^{\dagger}$, F. BOUCHON ${ }^{\dagger}$, Ch. DESCROZAILLES, P. FRIEZ ${ }^{\dagger}$, F. BROCHERE ${ }^{\dagger}$, A. RICHARD ${ }^{\dagger}$, R.-L. NINET ${ }^{\dagger}$, M. CHATEAU ${ }^{\dagger}$, P. COUSTAING ${ }^{\dagger}, A$. MUGNIER ${ }^{\dagger}$, R. WEILL', C. CREPY, J. OUVRARD ${ }^{\dagger}$, Y. COMMISSIONAT, B. DANGY ${ }^{\dagger}$, F. GARLOPEAU ${ }^{\dagger}$, J.-L. DEPHILIPPE', J. VIGNEUL, H. PETIT, M. BENOIST ${ }^{\dagger}$, G. FICHELLE' J. LAUFER, J.-P. SANTORO, P. LAUDENBACH ${ }^{+}$, P. DARGENT ${ }^{\dagger}$, Cl. SCHUHMANN, F. MAESTRONI ${ }^{\dagger}$, J.-P. DEFFEZ, H. OUVRARD, D. RIGOLET' ${ }^{\dagger}$, J. BUQUET, $\begin{array}{ll}\text { J.-P. RAGOT } & \\ & \text {, R. BUGUGNANI, P. BORDAIS, }\end{array}$ J.-M. LAURICHESSE', J.-Ch. BERTRAND, J.-F. LEGRAND, G. PASQUET, J. DICHAMP, D. BUCH， A. DEBOISE', C. BOZON, F. LARCHÉ, M. AMORIC, G. PRINC, V. TRAVERT, J.-P. LÉZY, F. LEVAVASSEUR, E. FAVRE, H. MISSISTRANO, T. PIRAL, C. BONNEFOY

\section{BULLETIN D'ADHÉSION À L'A.E.O.S.}

Oui, je souhaite devenir membre de I'A.E.O.S. Ci-joint le règlement de ma cotisation annuelle $2012: 20 €$ au lieu de 60 (offre réservée aux nouveaux abonnés des A.O.S.).

Je règle par chèque à l'ordre de l'A.E.O.S.

Dr:

Prénom :

Adresse

Code postal :

Ville :

Téléphone :

Fax :

Bulletin à retourner à : l'Association d'Enseignement d'Odontologie et de Stomatologie (A.E.O.S.) 179, rue Saint-Honoré - 75001 Paris - Tél. 0142605032 - Fax 0147034643 